\title{
Langerhans cell histiocytosis: An enigmatic disease
}

\section{Anubha Jain, Sushil Kumar, Priyanka Aggarwal, Mohan Kumar,Vineeta Gupta}

\begin{abstract}
Background: Langerhans cell histiocytosis (LCH) is a poorly understood disease with heterogeneous clinical presentation ranging from unifocal bony involvement to disseminated disease with life-threatening complications. Materials and Methods: The clinical profile, laboratory findings, treatment, and long-term outcome were retrieved from maintained medical records from January 2006 to January 2016 and were retrospectively analyzed.The extent of the disease was classified as per the LCH-III trial of "The Histiocyte Society." The assessment and categorization of treatment response followed LCH III trial definitions. Results: A total of 28 children with LCH were diagnosed. The age ranged between 5 months and 9 years, with a mean of $31 / 2$ years. The M: F ratio was 3:I. Single system, unifocal and multifocal bone diseases were seen in nine (32.1\%) and two (7.1\%) cases, respectively. Disseminated disease without risk organ involvement was seen in six (2I.I\%), whereas disseminated disease with risk organ involvement was seen in II (39.3\%) cases. The most common presentation was bony involvement (19 [67.8\%]), out of which $16(88.8 \%)$ had skull involvement. During follow-up, 17 (60.7\%) were in complete remission though five (17.8\%) of them relapsed, but achieved second remission. Two (7.1\%) were lost to follow-up. Six (21.4\%) had progressive disease of which four expired and two abandoned treatment. Two (10.7\%) refused the initiation of treatment. Conclusion: A better understanding of the disease, early suspicion, and diagnosis can improve the outcome of patients with $\mathrm{LCH}$.
\end{abstract}

Key words: Histiocytosis, Langerhans cell, outcome, risk organ, single system, survival

\section{Introduction}

Histiocytosis syndromes of childhood constitute a diverse group of disorders and are grouped together because of the common feature of proliferation of cells of monocytemacrophage system of bone marrow origin. It is classified on the basis of histopathological findings into two common forms - "Langerhans cell histiocytosis" (LCH) and "hemophagocytic lymphohistiocytosis." LCH was previously known as "Histiocytosis X" which included eosinophilic granuloma, Hand-Schuller-Christian disease, and Letterer-Siwe disease.

The hallmark of LCH is the presence of clonal proliferation of cells of the monocyte lineage containing the characteristic electron microscopic finding of Langerhans cells. This is a tennis racket-shaped bilamellar granule called Birbeck granule, seen in the cytoplasm of lesional cell in LCH. This granule expresses Langerin CD 207 and shows CD1a positivity which is now used for the definitive diagnosis of $\mathrm{LCH}$, and the demonstration of Birbeck granule is no longer necessary to establish diagnosis.

LCH remains a difficult-to-diagnose and a poorly understood disease. It has heterogeneous clinical presentation ranging from unifocal bony involvement to disseminated disease with life-threatening complications. The outcome of the disease also varies from spontaneous resolution to a progressive multisystem (MS) disorder with organ dysfunction. We present our experience of diagnosing and managing children with LCH over a decade at a single institution.

\section{Materials and Methods}

A total of 28 children of $\mathrm{LCH}$, managed in a pediatric oncology unit in North India from January 2006 to January 2016, were retrospectively analyzed.

The clinical profile, laboratory findings, treatment, and long-term outcome were retrieved from medical records

\begin{tabular}{|l|}
\hline Access this article online \\
\hline Quick Response Code: \\
\\
Website: www.sajc.org \\
\hline DOI: $10.4103 /$ sajc.sajc_211_18 \\
\hline
\end{tabular}
Hematology-Oncology, Institute of Medical Sciences, Banaras Hindu University, Varanasi, Uttar Pradesh, India Correspondence to: Dr.Anubha Jain,

E-mail: anubhajain 15@gmail.com maintained in the unit. A presumptive diagnosis based on morphological characteristics was made in majority of the suspected patients, and supplemental stains for S-100 protein/ CD1a antigen were done for definitive diagnosis.

Depending on the extent and localization of the disease at the time of evaluation, the following clinical categories as per the LCH III trial of "The Histiocyte Society" ${ }^{[1]}$ were defined:

1. Single-system LCH (SS-LCH): One organ/system involved (uni- or multi-focal): Bone: unifocal (single bone) or multifocal ( $>1$ bone), skin, lymph node (not the draining lymph node of another LCH lesion), lungs, hypothalamic-pituitary/central nervous system (CNS), and other (e.g. thyroid, thymus)

2. MS-LCH (MS-LCH): Two or more organs/systems involved with or without the involvement of "risk organs."

Children with both localized disease and disseminated disease were treated with induction chemotherapy consisting of weekly vinblastine (VBL) $\left(6 \mathrm{mg} / \mathrm{m}^{2}\right)$ for 6 to 8 weeks along with oral prednisolone $40 \mathrm{mg} / \mathrm{m}^{2} /$ day. Those who responded were shifted to maintenance chemotherapy consisting of 6MP, VBL, and prednisolone. Salvage protocol consisting of vincristine, etoposide, and cytosine arabinoside was given to those who did not respond to the initial induction chemotherapy. None of the patients underwent transplant during the study period.

\section{Assessment of treatment response}

The following definitions were applied to judge the effect of treatment:

- Nonactive disease: No evidence of disease (resolution of all signs or symptoms)

- Regressive disease: Regression of signs or symptoms

- Active disease: Stable disease: Persistence of signs or symptoms, no new lesions

- Progressive disease: Progression of signs and symptoms and/or appearance of new lesions.

This is an open access journal, and articles are distributed under the terms of the Creative Commons Attribution-NonCommercial-ShareAlike 4.0 License, which allows others to remix, tweak, and build upon the work non-commercially, as long as appropriate credit is given and the new creations are licensed under the identical terms.
For reprints contact: reprints@ medknow.com

How to cite this article: Jain A, Kumar S, Aggarwal P, Kumar M, Gupta V. Langerhans cell histiocytosis: An enigmatic disease. South Asian J Cancer 2019;8:183-5. 


\section{Definition of response criteria}

There are three categories of response:

- Better: Complete resolution/regression of disease

- Intermediate: Mixed/stable disease

- Worse: Progression of disease.

In isolated bone disease, progression was defined as the appearance of new bony lesions or lesions in other organs.

Follow-up of the patients ranged from 6 months to 168 months, with a median of 66 months.

\section{Results}

Age of the patients ranged between 5 months and 9 years, with a mean of $3 \frac{1}{2}$ years. Five patients $(17.8 \%)$ were equal to or less than 1 year of age at presentation. The M: F ratio was 3:1.

$\mathrm{SS}$, unifocal and multifocal bone diseases were seen in nine $(32.1 \%)$ and two $(7.1 \%)$ cases, respectively. Disseminated disease without risk organ involvement was seen in six $(21.1 \%)$, whereas disseminated disease with risk organ involvement was seen in $11(39.3 \%)$ of cases.

The presenting signs and symptoms are summarized in Table 1 where bony involvement was the most common presentation $(67.8 \%)$ followed by hepatosplenomegaly and/or lymphadenopathy in $42.8 \%$ each. Lung involvement was seen in three $(10.7 \%)$ patients, whereas another three $(10.7 \%)$ had central diabetes insipidus. One of these patients presented with only central diabetes insipidus initially and developed scalp swelling 6 months later. Seven children (25\%) had skin and/or nail involvement in the form of seborrhoeic dermatitis and dystrophic nails. The distribution of bony lesions is summarized in Table 2. Nineteen (67.8\%) children had bony lesions, out of which $16(88.8 \%)$ had skull involvement. Vertebrae was the next common site (26.3\%) followed by upper-limb bones, lower-limb bones, and pelvis (15.7\% each).

During follow-up, $17(60.7 \%)$ children were in complete remission (CR) though five (17.8\%) of them relapsed, but achieved second remission and continue to be in CR. Two (7.1\%) patients achieved remission, but were lost to follow-up after 1 year of treatment. Six (21.4\%) children had progressive disease, of which four expired and two abandoned treatment. Two $(10.7 \%)$ patients refused treatment initiation,

Table 1: Symptoms/signs of Langerhans cell histiocytosis

\begin{tabular}{lc}
\hline Signs and symptoms & $n(\%)$ \\
\hline Fever & $14(50)$ \\
Bone involvement & $19(67.8)$ \\
Liver/spleen involvement & $12(42.8)$ \\
Lymphadenopathy & $12(42.8)$ \\
Skin involvement & $7(25)$ \\
Lungs involvement & $3(10.7)$ \\
Diabetes insipidus & $3(10.7)$ \\
\hline
\end{tabular}

Table 2: Distribution of bone lesions in Langerhans cell histiocytosis $(n=19)$

\begin{tabular}{lc}
\hline Bone lesion distribution & $n(\%)$ \\
\hline Skull & $17(89.4)$ \\
Upper-limb bones & $3(15.7)$ \\
Lower-limb bones & $2(10.5)$ \\
Ribs & $2(10.5)$ \\
Vertebrae & $5(26.3)$ \\
Pelvis & $2(10.5)$ \\
\hline
\end{tabular}

whereas one patient chose to be treated at another center. All deaths occurred in patients with MS disease. None of the patients underwent stem cell transplantation during the study period.

\section{Discussion}

$\mathrm{LCH}$ is an uncommon and poorly understood disease of variable presentation. Patients with $\mathrm{LCH}$ can present to physicians of different specialties with variable complaints. They can present to an orthodontist with floating teeth, ${ }^{[2]}$ to a dermatologist with skin lesions, ${ }^{[3]}$ to an orthopedic surgeon with bony lesions, or to a pediatric surgeon with scalp swelling. ${ }^{[4]}$ One of our patients had only skin involvement and presented to the dermatologist. Diagnosis was established after a skin biopsy. Similarly, another child presented to dental outpatient department with loose teeth, in which an X-ray of the jaw showed several floating teeth, giving rise to the suspicion of LCH. A high index of suspicion is required to make an accurate diagnosis.

$\mathrm{LCH}$ can occur at any age, but younger age is more common. In our study, the mean age was $3 \frac{1}{2}$ years, and approximately one-fifth of the patients were infants. There was a male predominance with a $\mathrm{M} / \mathrm{F}$ ratio of $3: 1$. MS disease which constituted the bulk of the disease was found in 17 (60.7\%) cases. Another study has also reported the same in a tertiary care center. ${ }^{[5]}$ Bony involvement was the most common presentation followed by fever and lymphadenopathy. Similar findings have been reported in other studies. ${ }^{[6,7]}$ Pulmonary involvement was seen in $10.7 \%$ of our patients, whereas another study ${ }^{[8]}$ reported pulmonary $\mathrm{LCH}$ in $25 \%$ of patients. Three $(10.7 \%)$ patients had diabetes insipidus at the time of diagnosis. One of these patients presented with only diabetes insipidus and developed a boggy swelling over the scalp 6 months later which turned out to be LCH. In a series of forty cases from another center, ${ }^{[6]}$ three patients $(7.5 \%)$ had diabetes insipidus.

$\mathrm{LCH}$ has a very variable course akin to its presentation. The disease may have a very benign course requiring minimal treatment or may run a malignant course requiring second-line treatment. Nonresponding patients may require stem cell transplantation. For past several decades, it has been debated if the disease is primarily an immune dysregulatory disorder or a neoplasm. Over the years, evidence has provided the view that $\mathrm{LCH}$ is a neoplasm, and consequently recurrent episodes of the disease should be called relapse rather than reactivation. In our study also, several patients had relapse of the disease and achieved second CR. However, one-fifth of the patients had a progressive course, of which many succumbed to the disease and few abandoned treatment in view of poor response. All these patients had MS disease with or without risk organ involvement. Another study has also reported that event-free survival for MS-LCH with risk organ involvement is suboptimal in Indian patients. ${ }^{[9]}$ A couple of recent studies have reported good results in relapsed/refractory $\mathrm{LCH}$ and given new hope in this difficult subset of patients. However, patients with refractory/progressive disease in our series had adverse outcome. ${ }^{[10-12]}$

\section{Conclusion}

$\mathrm{LCH}$ is a rare disease and should be considered in patients with unexplained clinical manifestations of liver, lung, South Asian Journal of Cancer • Volume 8 •Issue 3 •uly-September 2019 
CNS, skin, and bone. Its myriad manifestations may further complicate and delay the diagnosis. Treatment and prognosis depends on the extent of the disease. Early recognition, diagnosis, and multidisciplinary approach can improve the outcome.

\section{Financial support and sponsorship}

Nil.

\section{Conflicts of interest}

There are no conflicts of interest.

\section{References}

1. Histiocyte Society. Langerhans cell histiocytosis: Evaluation and Treatment Guidelines. Histiocyte Society; April, 2009.

2. Bansal M, Srivastava VK, Bansal R, Gupta V, Bansal M, Patne S, et al. Severe periodontal disease manifested in chronic disseminated type of Langerhans cell histiocytosis in a 3-year old child. Int J Clin Pediatr Dent 2014;7:217-9.

3. Gupta V, Bansal M. Cutaneous Langerhans cell histiocytosis. Indian Pediatr 2012;49:79.

4. Bhat S, Nazir P, Bashir H, Reshi R, Sheikh S, Wani R, et al. Clinicopathological pattern of cranial unifocal Langerhans cell histiocytosis: A study at medical college hospital. Indian J Med Paediatr Oncol 2015;36: 183-5.
5. Bansal D, Marwaha RK, Trehan A, Gupta V, Varma N. Langerhans' cell histiocytosis: Experience from a single center. Indian Pediatr 2008;45:685-8.

6. Singh T, Satheesh CT, Appaji L, Aruna Kumari BS, Mamatha HS, Giri GV, et al. Langerhan's cell histiocytosis: A single institutional experience. Indian J Med Paediatr Oncol 2010;31:51-3.

7. Narula G, Bhagwat R, Arora B, Banavali S, Pai S, Nair C, et al. Clinico-biologic profile of Langerhans cell histiocytosis: A single institutional study. Indian J Cancer 2007;44:93-8.

8. Uppal P, Bothra M, Seth R, lyer V, Kabra SK. Clinical profile of Langerhans cell histiocytosis at a tertiary centre: A prospective study. Indian J Pediatr 2012;79:1463-7.

9. Totadri S, Bansal D, Trehan A, Srinivasan R, Varma N, Kakkar N, et al. The 5-year EFS of multisystem LCH with risk-organ involvement is suboptimal: A single-center experience from India. J Pediatr Hematol Oncol 2016;38:e1-5.

10. Kusuma Kumary P, Priyakumari T, Chellam VG, James FV, Nair MK. Langerhans cell histiocytosis in children less than 2 years of age. Indian Pediatr 1999;36:29-36.

11. Dua V, Yadav SP, Sachdeva A. Treatment of relapsed/refractory Langerhans cell histiocytosis: A single centre experience. Indian J Pediatr 2014;81:315-6.

12. Uppuluri R, Ramachandrakurup S, Subburaj D, Bakane A, Raj R. Excellent remission rates with limited toxicity in relapsed/refractory Langerhans cell histiocytosis with pulse dexamethasone and lenalidomide in children. Pediatr Blood Cancer 2017;64:110-2. 